\title{
Effects of colchicine on renal fibrosis and apoptosis in obstructed kidneys
}

\author{
Sejoong Kim ${ }^{1,2}$, Eun Sook Jung ${ }^{1}$, Jeonghwan $\mathrm{Lee}^{3}$, Nam Ju Heo ${ }^{1,4}, \mathrm{Ki}_{\text {Young } \mathrm{Na}^{1,2} \text {, and Jin Suk Han }}^{1}$
}

${ }^{1}$ Department of Internal Medicine, Seoul National University College of Medicine, Seoul; ${ }^{2}$ Department of Internal Medicine, Seoul National University Bundang Hospital, Seongnam; ${ }^{3}$ Department of Internal Medicine, Hallym University Hangang Sacred Heart Hospital, Seoul; ${ }^{4}$ Department of Internal Medicine, Seoul National University Hospital Healthcare System Gangnam Center, Seoul, Korea

Received: April 18, 2016 Revised : July 14, 2016 Accepted: July 16, 2016

\section{Correspondence to}

Jin Suk Han, M.D.

Department of Internal

Medicine, Seoul National

University Hospital, 101

Daehak-ro, Jongno-gu, Seoul

03080, Korea

Tel: +82-2-2072-2392

Fax: $+82-2-741-4876$

E-mail:jshan@snu.ac.kr
Background/Aims: Colchicine is an established drug for microtubule stabilization that may reduce tissue injury. No data were available that its effects may depend on the dosage of colchicine. We investigated the anti-fibrotic and apoptotic effects of various dose of colchicine in a unilateral ureteral obstruction (UUO) model.

Methods: Thirty-six Sprague-Dawley rats were randomly assigned into six groups. Two sham groups were divided into a vehicle-treated or colchicine-treated group $(100 \mu \mathrm{g} / \mathrm{kg} /$ day). Four UUO groups were treated with either vehicle or three different doses of colchicine for 7 days $(30,60$, and $100 \mu \mathrm{g} / \mathrm{kg} /$ day, intraperitoneally). All of the animals were sacrificed on day 7 .

Results: Colchicine treatment diminished acetylated $\alpha$-tubulin and tumor growth factor- $\beta$ immunoreactivities in the cortical area of the 7 -day obstructed kidneys, which was in dose dependent manner. Colchicine attenuated tubulointerstitial damage and apoptosis in both cortical and medullary area, and beneficial effects of colchicine therapy were dramatically shown at the higher dosage of colchicine. The expression levels of cleaved caspase-3, ED-1, and fibronectin were decreased in UUO animals.

Conclusions: We found that the proper dosage of colchicine may have anti-fibrotic and anti-apoptotic effects in obstructed kidneys. For clinical applications, an optimal dose of colchicine should be evaluated to maximize the prevention of renal disease progression.

Keywords: Colchicine; Kidney; Microtubules; Ureteral obstruction

\section{INTRODUCTION}

As the number patients with chronic kidney disease rapidly grows, a need for novel and effective medication for renal disease is rising. Discovering unknown effects of established drugs will be another way to ameliorate the progression of renal disease. Colchicine is a microtubule-targeting agent which depolymerizes microtubules and blocks mitosis [1]. Colchicine is used as an anti-cancer medication for apoptosis induction in cancer cells. Additionally, it has previously been used to relieve acute gout flares. In contrast, some researchers have demonstrated that microtubule alteration using colchicine induces survival signals [2].

Colchicine has demonstrated its anti-fibrotic and anti-inflammatory effects in several kidney models of crescentic nephritis [3], chronic cyclosporine nephrotoxicity [4], diabetic nephropathy [5], and hypertensive chronic kidney disease [6]; however, these studies used only a single dosage of colchicine. The kidney has functional nephron heterogeneity, suggesting that the medulla is much more susceptible than the cortex to obstructive nephropathy and renal ischemia $[7,8]$. However, the majority of the previous studies did not address this dispar- 
ity between the cortex and medulla nor did they evaluate these areas separately.

Unilateral ureteral obstruction (UUO) is a good model of tubular apoptosis and interstitial fibrosis in the kidneys $[9,10]$. Mechanical stress induces the over-polymerization of microtubules, and contributes to tissue morphogenesis, remodeling, and fibrosis [11]. We investigated the microtubule-depolymerizing effects of various doses of colchicine on renal fibrosis and apoptosis at both the surface and deep nephron during obstructive nephropathy. Our findings provide a new perspective on the renoprotective effects of colchicine.

\section{METHODS}

The Institutional Animal Care and Use Committee (Seoul National University Bundang Hospital) approved the experimental protocol (No. BA1112-094/074-01).

Thirty-six specific pathogen-free male Sprague-Dawley rats ( 5 to 6 weeks old; Orient Bio Inc., Seongnam, Korea), initially weighing approximately $240 \mathrm{~g}$, were kept in a temperature-controlled room and were allowed free access to standard laboratory chow and tap water. UUO was performed by ligation of the left ureter and a sham operation was identical to the UUO operation except for the ligation of the ureter. A total of rats comprised the sham groups $(n=12)$ and the UUO groups $(n=24)$. The sham groups were divided into a vehicle-treated group and a colchicine-treated group $(100 \mu \mathrm{g} / \mathrm{kg} /$ day $)$; the UUO groups were divided in dose dependent manner (vehicle, 30,60 , and $100 \mu \mathrm{g} / \mathrm{kg} /$ day colchicine treatment). For 7 days, the rats in the vehicle group received a daily an intraperitoneal injection of only sterile saline, and the rats in the colchicine group received colchicine (Sigma-Aldrich Corp., Yongin, Korea) intraperitoneally on a daily basis for 7 days. On day 7 , all of the animals were sacrificed, and the kidneys were isolated and immediately stored at $-70^{\circ} \mathrm{C}$ until use.

Blocks of kidney were fixed in 10\% buffered formalin and embedded in paraffin for routine histological examination. The glomerulosclerosis index was assessed on periodic-acid Schiff-stained paraffin sections at a magnification of $\times 400$. The tubulointerstitial injury indexes were determined using a semiquantitative scoring system on Masson's trichrome-stained sections at a magnification of $\times 100$. More than 10 consecutive fields were examined and the grading was performed in a blind manner. The tubulointerstitial injury score was estimated based on the tubular atrophy, interstitial inflammation, fibrosis and casts in the range of o to 3 (o, no change; $1,<25 \% ; 2,25 \%$ to $50 \%$; and $3,50 \%$ to $100 \%$ ) [12]. Apoptosis was detected by enzymatically labeling DNA strand breaks using terminal deoxynucleotidyl transferase dUTP nick end labeling (TUNEL) staining. TUNEL staining was performed using a Cell Death Detection Kit (Roche, Mannheim, Germany). To reveal the total quantity of nuclei, the same slides were stained with 4',6'-diamidino-2-phenylindole and the TUNEL-positive cells were counted in at least 10 different fields under $\times 200$ magnification. Positive staining was detected using a laser-scanning confocal microscopy (Zeiss LSM 710, Carl Zeiss, Oberkochen, Germany). The kidneys were dissected into the cortex and inner stripe of the outer medulla and each region was separately homogenized using a tissue homogenizer (PowerGun 125, Fisher Scientific, Pittsburgh, PA, USA) [13]. Each protein analyzed using Pierce BCA Protein Assay Kit (Pierce Biotechnology, Rockford, IL, USA). The samples were fractionated by SDS-PAGE (sodium dodecyl sulfate polyacrylamide gel electrophoresis). After transfer onto the protran nitrocellulose transfer membrane (Whatman, Gassel, Germany), the blots were probed using the following antibodies: monoclonal mouse anti-acetylated $\alpha$-tubulin antibody (Abcam, Cambridge, UK) and anti- $\alpha$-tubulin (Santa Cruz Biotechnology, Santa Cruz, CA, USA), anti-vimentin (Santa Cruz Biotechnology), anti-E-cadherin (BD biosciences, San Jose, CA, USA), anti- $\alpha$-smooth muscle actin (Abcam), anti-fibronectin (F3648, Sigma-Aldrich, St. Louis, MO, USA), anti-caspase-3 (Cell Signaling Technology, Danvers, MA, USA), anti-caspase-9 (BD Biosciences), ED-1 (MCA1957, AbD serotec, Oxford, UK), and goat polyclonal anti- $\beta$-actin (Santa Cruz Biotechnology). Incubation with peroxidase-conjugated anti-rabbit or anti-goat IgG secondary antibodies (Pierce Biotechnology) was followed by band visualization using enhanced chemiluminescence (ECL RPN 2106, Amersham Pharmacia Biotech, Buckinghamshire, UK). The band densities were quantified by densitometry (GS-70o Imaging Densitometry, Bio-Rad, Hercules, CA, USA). Tumor growth factor $\beta 1$ (TGF- $\beta 1$ ) immunoassay system were purchased from Promega 

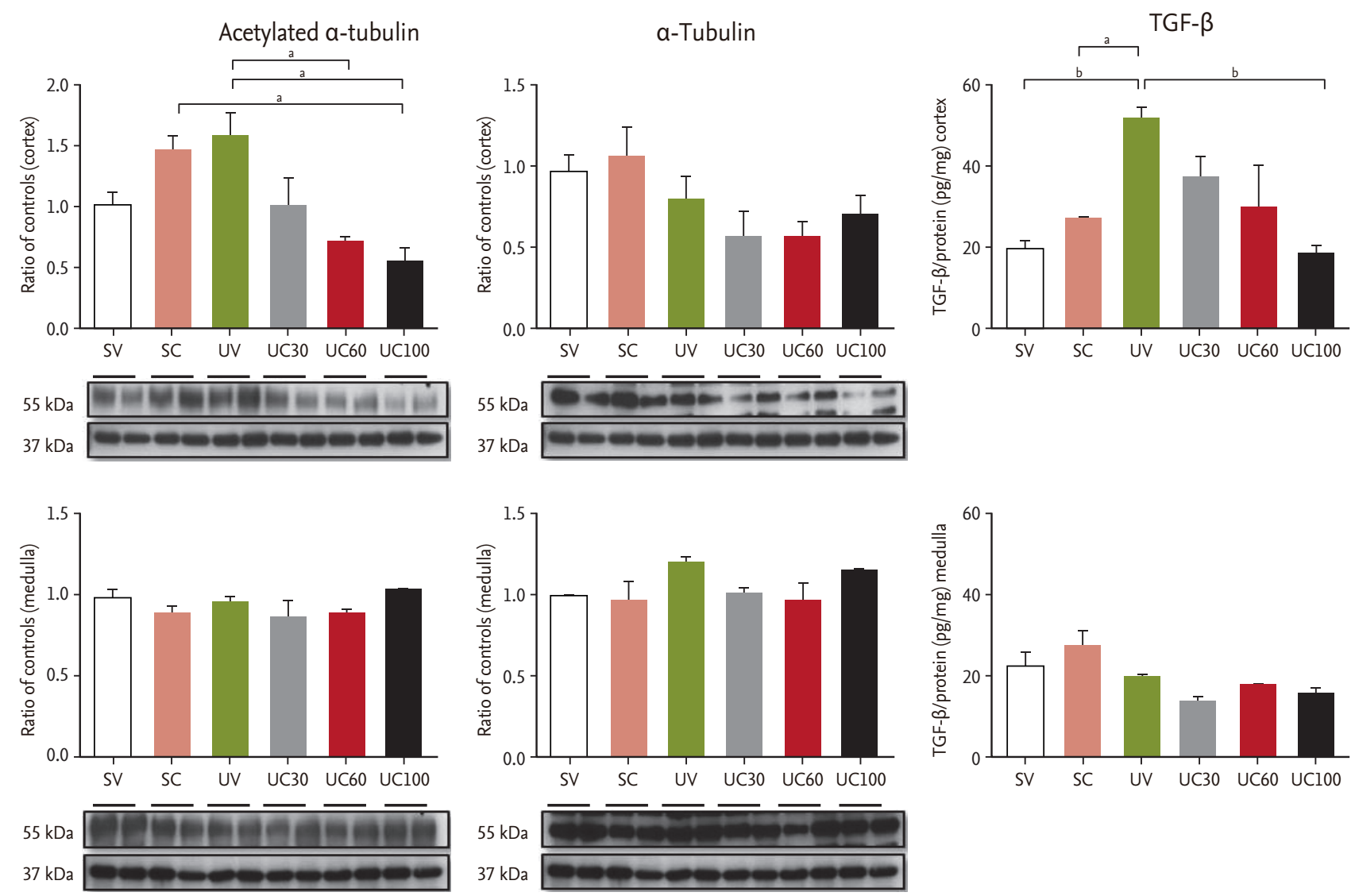

\section{A}

B

\section{C}

Figure 1. Comparison of (A) acetylated $\alpha$-tubulin, (B) $\alpha$-tubulin, and (C) tumor growth factor $\beta$ (TGF- $\beta$ ) using colchicine treatment in ureteral obstructive kidneys. The expression of acetylated $\alpha$-tubulin was increased in unilateral ureteral obstruction (UUO)-operated rats and was diminished in the cortical lesions of colchicine-treated UUO rats. The cortical expression of TGF- $\beta$ was increased after UUO, and then gradually decreased during colchicine treatment. SV, sham-operated rats with vehicle; SC, sham-operated rats with colchicine treatment; UV, ureteral obstructive rats with vehicle; UC, ureteral obstructive rats with colchicine treatment. ${ }^{\mathrm{a}} \mathrm{p}<0.05,{ }^{\mathrm{b}} \mathrm{p}<0.01$.

(Maison, WI, USA)

\section{Statistical analysis}

Comparisons between two groups were made with the Mann-Whitney $U$ test, and comparisons among three groups were made with the Kruskal-Wallis test (SPSS version 18.o, SPSS Inc., Chicago, IL, USA). Band density values were standardized by dividing them by the average value of the control group. The mean for the control group was defined as $100 \%$, and the results were expressed as the mean \pm SEM. A $p<0.05$ was considered statistically significant.

\section{RESULTS}

To evaluate the effects of colchicine on microtubule polymerization, immunoblots of acetylated $\alpha$-tubulin, $\alpha$-tubulin, and vimentin were performed. The immunoblotting of acetylated $\alpha$-tubulin in UUO-operated rats was slightly increased compared to the sham-operated rats and was diminished in the cortical lesion of the 60- and 100-mg colchicine-treated UUO rats (Fig. 1). The immunoreactivity of $\alpha$-tubulin was not significantly changed in colchicine- or vehicle-treated UUO rats. Although immunoblotting demonstrated that vimentin was also increased in the UUO models, colchicine treatment failed to exhibit differences in the UUO rats (data not shown). The cortical expres- 

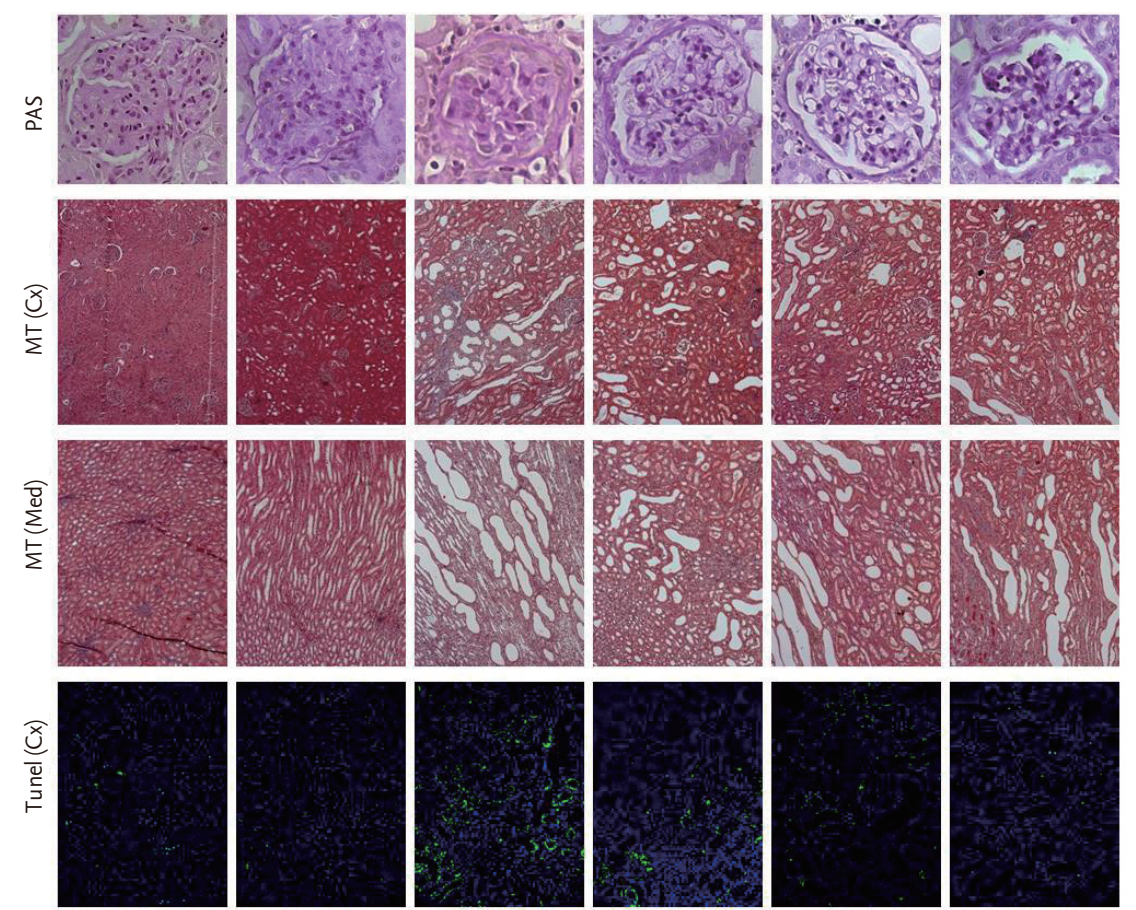

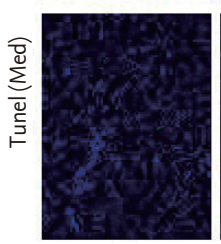

SV

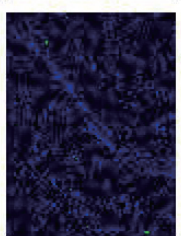

SC

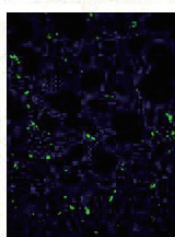

UV

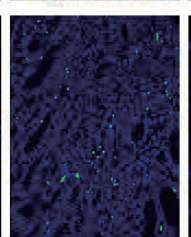

UC30

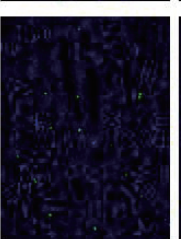

UC60
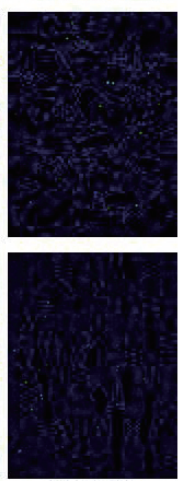

UC100
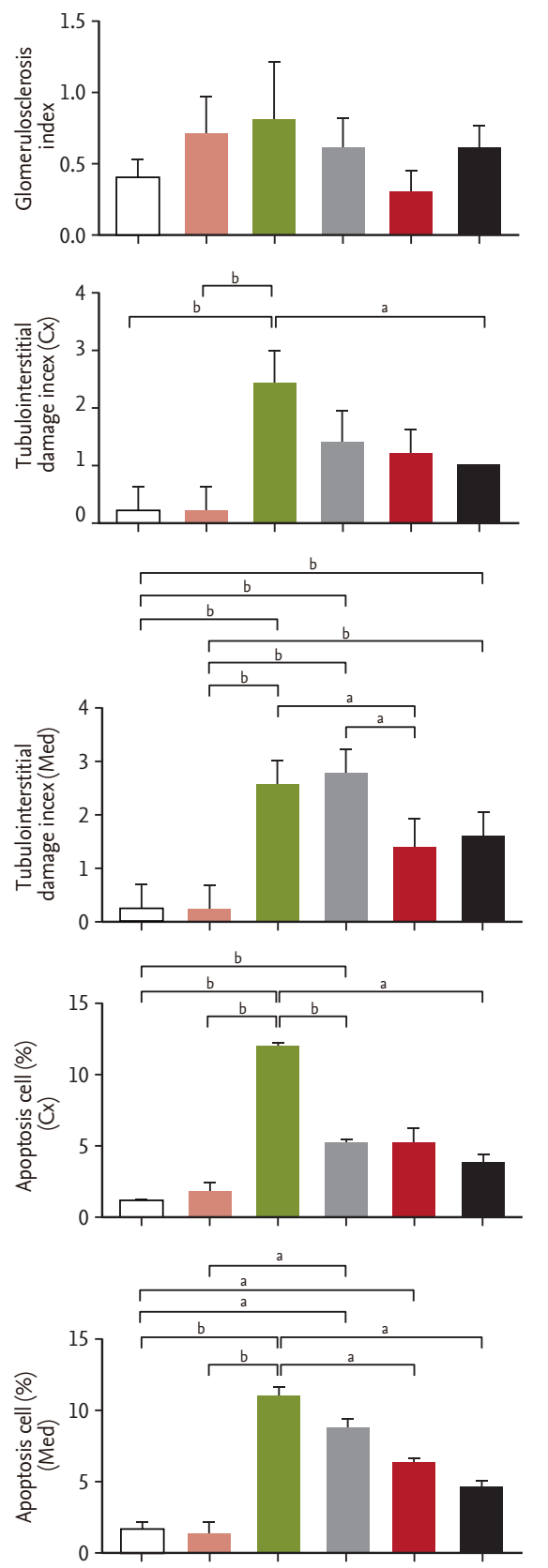

Figure 2. Pathologic findings in ureteral obstructive kidneys: glomerulosclerosis (periodic-acid Schiff [PAS] stain, $\times 400$ ), tubulointerstitial damage (Masson's-trichrome $[\mathrm{MT}]$ stain, $\times 100$ ), and apoptosis (terminal deoxynucleotidyl transferase dUTP nick end labeling [TUNEL] stain, $\times 200$ ). Tubulointerstitial damage and apoptosis were attenuated after colchicine treatment. Cx, cortical lesion; Med, medullary lesion; SV, sham-operated rats with vehicle; SC, sham-operated rats with colchicine treatment; UV, ureteral obstructive rats with vehicle; UC, ureteral obstructive rats with colchicine treatment. ${ }^{\mathrm{a}} \mathrm{p}<0.05,{ }^{\mathrm{b}} p<0.01$. odic-acid Schiff staining. The tubular damage and interstitial fibrosis scores as revealed by Masson's trichrome staining were increased in the UUO rats compared to the sham-operated rats, and these scores in both cortical and medullary lesions were attenuated in the high-dose colchicine-treated UUO rats to a greater degree compared to vehicle-treated UUO rats (Fig. 2). The number of tubular apoptotic cells was significantly increased after the UUO operation, but was significantly reduced in both cortical and medullary lesions with colchicine 

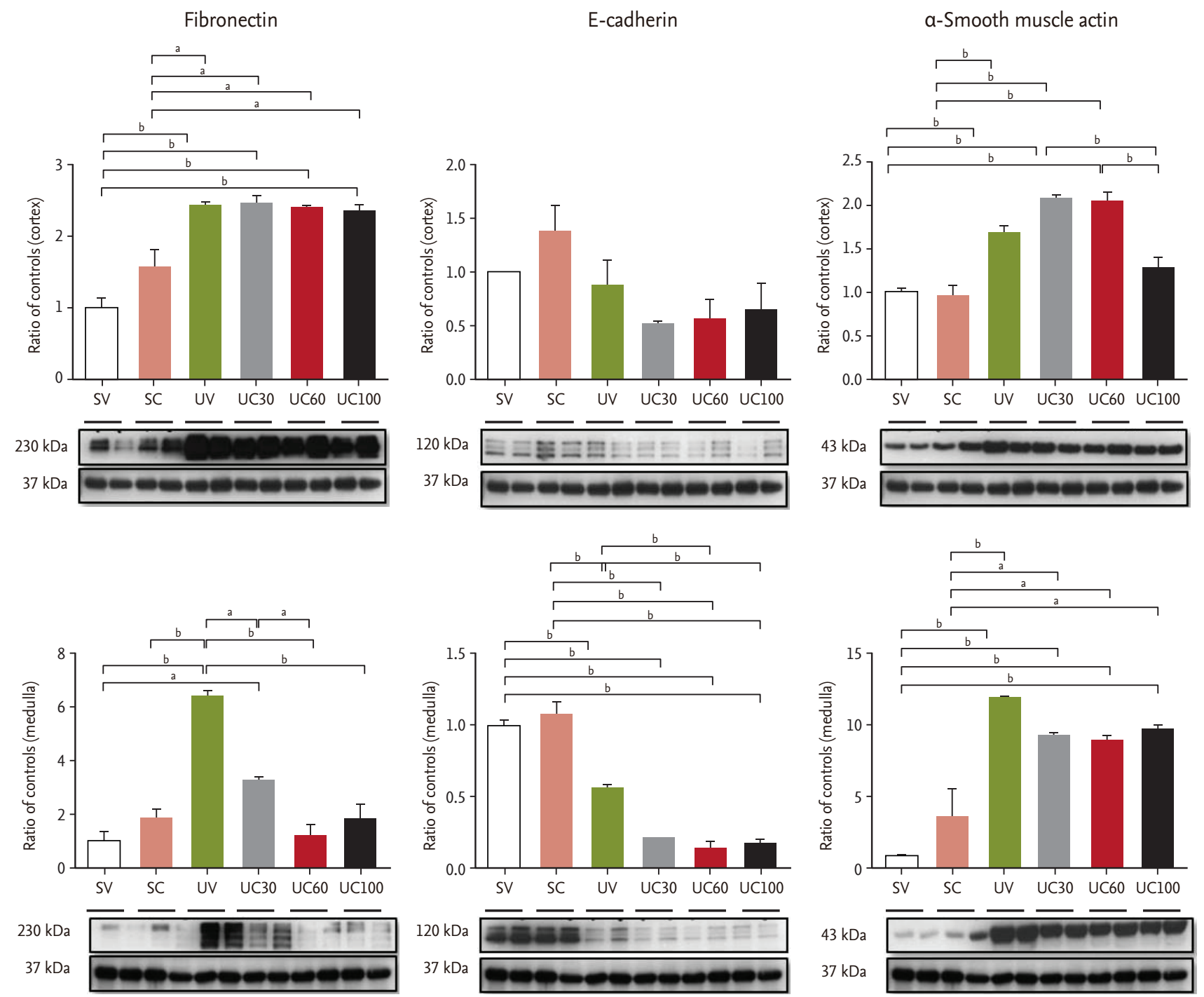

A

B

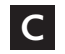

Figure 3. Immunoblots related to fibrosis: (A) fibronectin, (B) E-cadherin, and (C) $\alpha$-smooth muscle actin. Fibronectin expression was reduced with colchicine therapy; epithelial-mesenchymal transition markers were not changed. SV, sham-operated rats with vehicle; SC, sham-operated rats with colchicine treatment; UV, ureteral obstructive rats with vehicle; UC, ureteral obstructive rats with colchicine treatment. ${ }^{\mathrm{a}} \mathrm{p}<0.05,{ }^{\mathrm{b}} \mathrm{p}<0.01$.

treatment in a dose-dependent manner (Fig. 2).

Fibronectin was markedly increased in the UUO models, whereas it was significantly ameliorated with colchicine treatment in the medullary lesions of UUO rats (Fig. 3). The expression of E-cadherin decreased while $\alpha$-smooth muscle actin increased after ureteral obstruction; however, there were no differences between the colchicine- and vehicle-treated groups.

The expression of apoptosis-related protein, including cleaved caspase-3 and caspase-9, was examined. Cleaved caspase-3 was increased in UUO models and was abrogated in cortical and medullary lesions with increasing doses of colchicine (Fig. 4). The expression of caspase-9 was slightly decreased with colchicine treatment in the UUO rats, but no significant differences were found (Fig. 4). Immunoblots of ED-1 were also evaluated to quantify inflammation. In the UUO rats, the expression of ED-1 was increased compared to the sham-operated controls, whereas ED-1 expression was attenuated after colchicine treatment in the UUO rats, especially in medullary lesions (Fig. 4). 

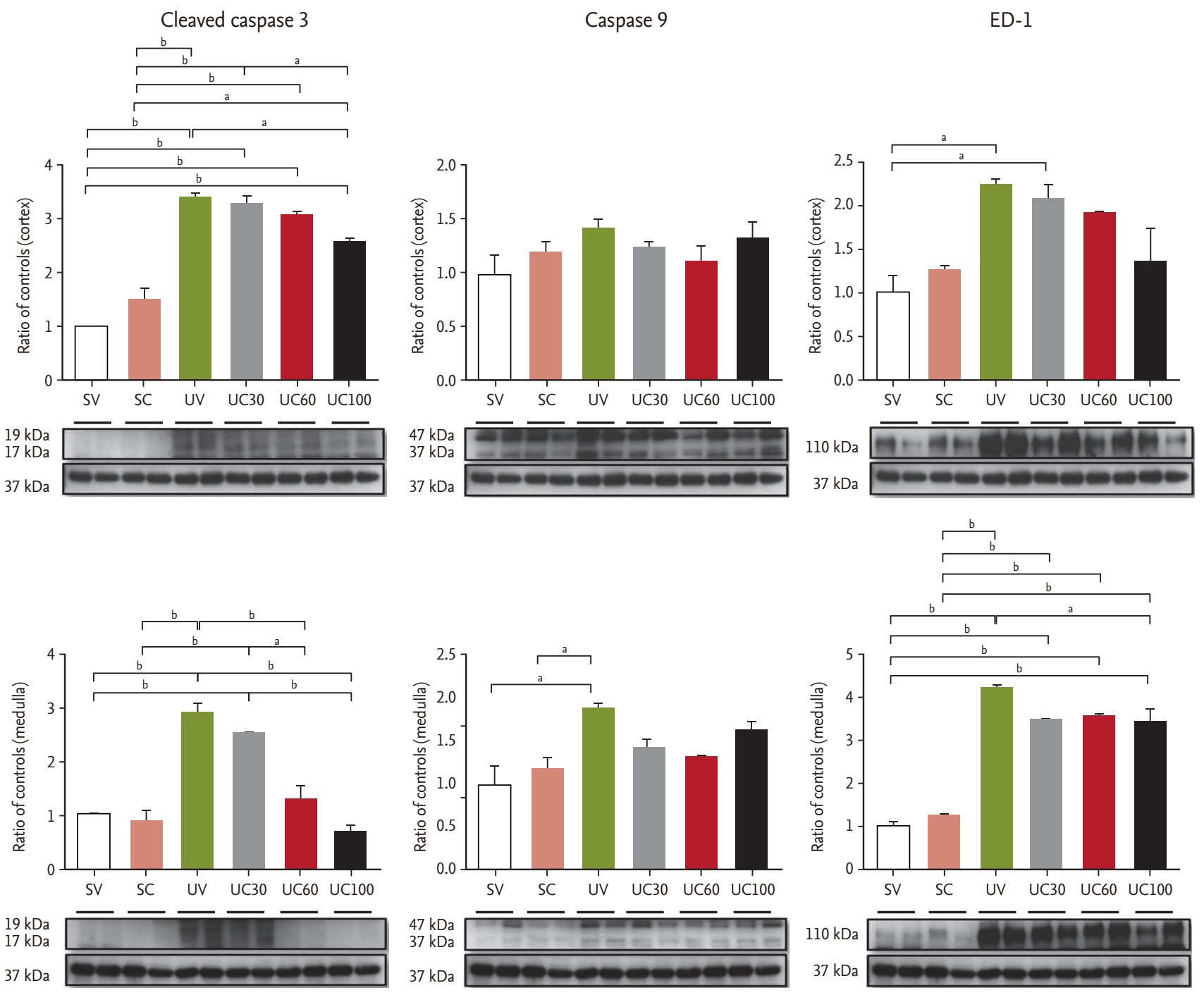

\section{A}

B

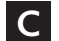

Figure 4. Immunoblots related to apoptosis and inflammation: (A) cleaved caspase-3, (B) caspase-9, and (C) ED-1. The caspase 3 pathway was blocked and ED-1 was slightly decreased with high-dose colchicine therapy. SV, sham-operated rats with vehicle; SC, sham-operated rats with colchicine treatment; UV, ureteral obstructive rats with vehicle; UC, ureteral obstructive rats with colchicine treatment. ${ }^{\mathrm{a}} \mathrm{p}<0.05,{ }^{\mathrm{b}} \mathrm{p}<0.01$.

\section{DISCUSSION}

We found that an appropriate dosage of colchicine can ameliorate renal fibrosis and apoptosis in obstructive nephropathy, indicating that colchicine can be used to prevent the progression of kidney injury. The depolymerizing effect of colchicine may lead to the attenuation of renal fibrosis and apoptosis in obstructed kidneys.

We found that ureteral obstruction may induce the over-activation and alteration of microtubules in cortical lesions. Exogenous mechanical forces contribute to the overexpression of acetylated tubulin [14]. Colchicine is known to inhibit the polymerization of tubulin [4] and the acetylation of tubulin polymers [15]. When we evaluated cytoskeletal components such as microtubules (acetylated $\alpha$-tubulin and $\alpha$-tubulin) and intermediate filaments (vimentin), we confirmed the decreased expression of acetylated $\alpha$-tubulin after treatment with colchicine in the UUO kidneys. TGF- $\beta$ plays a crucial role in the promotion of renal fibrosis, and colchicine suppresses TGF- $\beta$ expression and diminishes cyclosporine related nephrotoxicity $[16,17]$, which may be con- 
sistent with our results that colchicine treatment led to down-regulation of TGF- $\beta$ and reduced renal fibrosis. Some researchers have reported that the deacetylation of $\alpha$-tubulin by histone deacetylase 6 contributes to the TGF- $\beta$ related epithelial mesenchymal transition [18]. However, this is inconsistent with our finding that the attenuation of acetylated $\alpha$-tubulin by colchicine was associated with decreased TGF- $\beta$ expression. Acetylated $\alpha$-tubulin is present at the junction between a T-cell and an antigen presenting cell, and the deacetylation of tubulin may attenuate the lymphocyte activation signaling cascade [19]. This is consistent with our finding that ED-1, a cellular marker that is specific for macrophages, has been shown to be decreased by colchicine treatment in UUO models. It is possible that the attenuation of inflammatory cells may lead to reduced TGF- $\beta$ production, which may be associated with the anti-apoptotic and anti-fibrotic effects of colchicine treatment.

We found that high dose colchicine led to a reduction in acetylated $\alpha$-tubulin and caspase-3 expression in the cortical lesions of UUO rats. This is consistent with a previous report that found that the acetylation of $\alpha$-tubulin led to the activation of caspase-3 [4], which may be related to renal apoptosis [20]. In the current study, only caspase-3 expression and tubular apoptosis were attenuated in medullary lesions without microtubule alterations. The extracellular signal-regulated kinase (ERK) pathway may be involved in mechanical stress-induced hypertrophic responses [21]. The initial activation of ERK after UUO was restricted to the medullary collecting ducts and the thick ascending limb of Henle, which precedes tubular proliferation and injury [22]. In vitro studies showed that colchicine causes inactivation of ERK [21]. It could be possible that a microtubule independent anti-apoptotic effect of colchicine may occur in the medullary lesions of obstructed kidneys. Some studies demonstrated an apparent interplay between early apoptosis and subsequent fibrosis, indicating that apoptosis may be an early event that precedes the onset of fibrosis [23]. Although we failed to show that colchicine prevents the epithelial mesenchymal transition, a colchicine-related anti-apoptotic effect may ameliorate kidney fibrosis. We showed that fibronectin expression was significantly ameliorated with colchicine treatment in medullary lesions. This finding may be consistent with data that show the effect of colchicine on collagen accumulation by inhibiting fibronectin translation and expression $[6,24]$.

Fibronectin expression was decreased after colchicine treatment in UUO kidneys, which is consistent with previous reports [5]. In contrast, $\alpha$-smooth muscle actin expression was not changed after colchicine treatment. $\alpha$-smooth muscle actin is normally expressed in the smooth muscle lineages cells, particularly fibroblasts. Fibronectin is a glycoprotein of the extracellular matrix that is secreted by fibroblasts. This suggests colchicine treatment may inhibit the secretory function of fibroblasts, but may not completely inhibit the proliferation of fibroblasts in this UUO model. This study has some limitations. We did not measure renal function using parameters such as serum creatinine levels or urinary biomarkers. However, tissue injury indices are used in many UUO models as outcomes of treatment [25,26]. It is possible that colchicine may retard the exocytosis of intracellular vesicles such as those expressing aquaporin-2 and H+-ATPase via microtubule alterations that could be related to impaired urine concentration and tubular acidification [27,28]. Unfortunately, we did not evaluate the expression of those vesicles; this should be evaluated in the future. In addition, longer treatment with colchicine should be evaluated, as these data were limited to 7-day UUO models.

We found that colchicine may have beneficial effects on renal apoptosis and fibrosis in obstructed kidneys. For clinical applications, an appropriate dose of colchicine should be evaluated to maximize the prevention of renal disease progression.

\section{KEY MESSAGE}

1. Colchicine has anti-fibrotic and anti-apoptotic effects in the obstructed kidneys.

2. Those beneficial effects may depend on the dosage of colchicine.

3. For clinical applications, an optimal dose of colchicine should be evaluated to maximize the prevention of renal disease progression.

\section{Conflict of interest}

No potential conflict of interest relevant to this article was reported. 


\section{Acknowledgments}

This work was supported by a grant No. 2013 R1A1A1012689 from the National Research Foundation of Korea, and a grand of the Korea Health Technology R\&D Project through the Korea Health Industry Development Institute (KHIDI), funded by the Ministry of Health and Welfare, Republic of Korea (grant number: HI16C2221).

\section{REFERENCES}

1. Kim SK, Cho SM, Kim H, et al. The colchicine derivative CT20126 shows a novel microtubule-modulating activity with apoptosis. Exp Mol Med 2013;45:e19.

2. Mollinedo F. Survival and apoptotic signals in the action of microtubule-targeting antitumor drugs. IDrugs 2005;8:127-143.

3. McClurkin C Jr, Phan SH, Hsu CH, et al. Moderate protection of renal function and reduction of fibrosis by colchicine in a model of anti-GBM disease in the rabbit. J Am Soc Nephrol 1990;1:257-265.

4. Li C, Yang CW, Ahn HJ, et al. Colchicine decreases apoptotic cell death in chronic cyclosporine nephrotoxicity. J Lab Clin Med 2002;139:364-371.

5. Li JJ, Lee SH, Kim DK, et al. Colchicine attenuates inflammatory cell infiltration and extracellular matrix accumulation in diabetic nephropathy. Am J Physiol Renal Physiol 2009;297:F200-F209.

6. Guan T, Gao B, Chen G, et al. Colchicine attenuates renal injury in a model of hypertensive chronic kidney disease. Am J Physiol Renal Physiol 2013;305:F1466-F1476.

7. Wilson DR. Nephron functional heterogeneity in the postobstructive kidney. Kidney Int 1975;7:19-26.

8. Mason J, Welsch J, Takabatake T. Disparity between surface and deep nephron function early after renal ischemia. Kidney Int 1983;24:27-36.

9. Gobe GC, Axelsen RA. Genesis of renal tubular atrophy in experimental hydronephrosis in the rat. Role of apoptosis. Lab Invest 1987;56:273-281.

10. Truong LD, Choi YJ, Tsao CC, et al. Renal cell apoptosis in chronic obstructive uropathy: the roles of caspases. Kidney Int 2001;60:924-934.

11. Yutao X, Geru W, Xiaojun B, Tao G, Aiqun M. Mechanical stretch-induced hypertrophy of neonatal rat ventricular myocytes is mediated by beta(1)-integrin-microtubule signaling pathways. Eur J Heart Fail 2006;8:16-22.

12. Kim S, Heo NJ, Jung JY, et al. Changes in the sodium and potassium transporters in the course of chronic renal failure. Nephron Physiol 2010;115:p31-p41.

13. Na KY, Kim GH, Joo KW, et al. Chronic furosemide or hydrochlorothiazide administration increases H+-ATPase B1 subunit abundance in rat kidney. Am J Physiol Renal Physiol 2007;292:F1701-F1709.

14. Gourlay CW, Ayscough KR. The actin cytoskeleton: a key regulator of apoptosis and ageing? Nat Rev Mol Cell Biol 2005;6:583-589.

15. Jung HI, Shin I, Park YM, Kang KW, Ha KS. Colchicine activates actin polymerization by microtubule depolymerization. Mol Cells 1997;7:431-437.

16. Disel U, Paydas S, Dogan A, Gulfiliz G, Yavuz S. Effect of colchicine on cyclosporine nephrotoxicity, reduction of TGF-beta overexpression, apoptosis, and oxidative damage: an experimental animal study. Transplant Proc 2004;36:1372-1376.

17. El-Sakka AI, Bakircioglu ME, Bhatnagar RS, Yen TS, Dahiya R, Lue TF. The effects of colchicine on a Peyronie's-like condition in an animal model. J Urol 1999;161:1980-1983.

18. Shan B, Yao TP, Nguyen HT, et al. Requirement of HDAC6 for transforming growth factor-beta1-induced epithelial-mesenchymal transition. J Biol Chem 2008;283:2106521073.

19. Serrador JM, Cabrero JR, Sancho D, Mittelbrunn M, Urzainqui A, Sanchez-Madrid F. HDAC6 deacetylase activity links the tubulin cytoskeleton with immune synapse organization. Immunity 2004;20:417-428.

20. Ruwhof C, van der Laarse A. Mechanical stress-induced cardiac hypertrophy: mechanisms and signal transduction pathways. Cardiovasc Res 2000;47:23-37.

21. Stone AA, Chambers TC. Microtubule inhibitors elicit differential effects on MAP kinase (JNK, ERK, and p38) signaling pathways in human KB-3 carcinoma cells. Exp Cell Res 2000;254:110-119.

22. Masaki T, Foti R, Hill PA, Ikezumi Y, Atkins RC, Nikolic-Paterson DJ. Activation of the ERK pathway precedes tubular proliferation in the obstructed rat kidney. Kidney Int 2003;63:1256-1264.

23. Tao Y, Kim J, Faubel S, et al. Caspase inhibition reduces tubular apoptosis and proliferation and slows disease progression in polycystic kidney disease. Proc Natl Acad Sci U S A 2005;102:6954-6959.

24. Zhou B, Rabinovitch M. Microtubule involvement in 
translational regulation of fibronectin expression by light chain 3 of microtubule-associated protein 1 in vascular smooth muscle cells. Circ Res 1998;83:481-489.

25. Park SH, Choi MJ, Song IK, et al. Erythropoietin decreases renal fibrosis in mice with ureteral obstruction: role of inhibiting TGF-beta-induced epithelial-to-mesenchymal transition. J Am Soc Nephrol 2007;18:1497-1507.

26. Chevalier RL, Forbes MS, Thornhill BA. Ureteral obstruc- tion as a model of renal interstitial fibrosis and obstructive nephropathy. Kidney Int 2009;75:1145-1152.

27. Kwon TH, Frokiaer J, Nielsen S. Regulation of aquaporin-2 in the kidney: a molecular mechanism of body-water homeostasis. Kidney Res Clin Pract 2013;32:96-102.

28. Kang SH, Kim J, Park JW. Biopsy-proven type 1 renal tubular acidosis in a patient with metabolic acidosis. Korean J Intern Med 2012;27:119. 